\title{
Ability of Orally Administered IFN- $\alpha 4$ to Inhibit Naturally Occurring Gingival Inflammation in Dogs
}

\author{
Akira ITO ${ }^{1,3)}$, Emiko ISOGAI ${ }^{2)}$, Kuniaki YOSHIOKA ${ }^{3)}$, Ko SATO ${ }^{3)}$, Naomi HIMENO ${ }^{3)}$ and Toru GOTANDA ${ }^{3) *}$ \\ ${ }^{1)}$ Research Institute of Genome-Based Biofactory, National Institute of Advanced Industrial and Science Technology (AIST), Sapporo, \\ Hokkaido 062-8517, 2) Department of Preventive Dentistry, Health Sciences University of Hokkaido, Ishikari-Tobetsu, Hokkaido 061- \\ 0293 and ${ }^{3)}$ Research Center for Biologicals, The Kitasato Institute, Kitamoto, Saitama 364-0026, Japan
}

(Received 12 May 2009/Accepted 2 April 2010/Published online in J-STAGE 16 April 2010)

\begin{abstract}
It has been reported that type I interferons (IFN- $\alpha / \beta$ ) play an important role in innate immune responses against viral and bacterial infections. In this study, we used and examined naturally occurred canine periodontal disease to show the therapeutic efficacy of low dose oral administration (LDOA) of canine IFN- $\alpha$ subtype 4 (CaIFN- $\alpha 4)$. We administered purified recombinant CaIFN- $\alpha 4$ expressed in a baculovirus system to dogs with or without gingival inflammation. We found that LDOA of CaIFN- $\alpha 4$ reduce periodontopathic bactrerial counts. LDOA induced improvement of naturally occurring gingival inflammation, and reduction of the stress marker responses was also observed after LDOA. These results suggest that LDOA of CaIFN- $\alpha 4$ has effectiveness for improvement of naturally occurring gingival inflammation in dogs.
\end{abstract}

KEY WORDS: canine, cytokine, interferon, periodontics, therapy.

J. Vet. Med. Sci. 72(9): 1145-1151, 2010

Periodontal disease is a multifactorial disease of the tissues supporting the teeth, and both host and bacterial factors influence the course of the disease [24]. The progression of periodontal disease is not clearly understood, but is characterized by the local accumulation of activated leukocytes [27]. It is likely that cytokines produced locally influence the development of this immune response [12].

Type I interferons (IFN- $\alpha / \beta$ ) were originally identified by their ability to prevent viral replication [1]. IFN- $\alpha$ is primarily produced by fibroblasts or type 1 helper T-cells upon stimulation by viral or bacterial infections [29]. The oral administration of IFN- $\alpha$ has been found to inhibit bacterial infections such as Listeria monocytogenes [26]. Thus, it has been suggested that IFN- $\alpha$ could provide immune control of disease interfering with bacterial growth as well as by enhancing the production of antibacterial immunoglobulin $\mathrm{G}(\mathrm{IgG})$ antibodies [25, 29].

Several studies have shown that type I IFNs, in addition to their classic antiviral and tumoricidal activities, also protect the host from nonviral pathogens and regulate systemic immune responses [3, 4]. Recently, it has been reported that low dose oral administration (LDOA) of type I IFN is effective against chronic diseases and infectious diseases $[2,6,7$, $13,14]$. The mechanism of systemic immune response regulation by LDOA of IFN- $\alpha$ is suggested as humoral or cellmediated via mucosal epithelial cells $[5,11,37,38,41]$. It is also suggested that neural cascade via IFN receptors of the oral and throat cavity is possibly involved [17]. Thus, type I IFNs are important in innate and adaptive immune responses, and can be effective in a broad range of clinical

* Correspondence to: Gotanda, T., Research Center for Biologicals, The Kitasato Institute, 6-111, Arai, Kitamoto-shi, Saitama 364-0026, Japan.

e-mail: tgotanda@insti.kitasato-u.ac.jp applications. Furthermore, it has been reported that the combination of decreased interleukin (IL)- 8 and decreased IFN- $\alpha$ concentrations at periodontal disease sites may reflect reduced antibacterial host defense activity [25].

It has been reported that canine periodontal disease is frequently found in dogs, and it has been estimated that approximately $80 \%$ of dogs demonstrate some degree of periodontal disease by 4 years of age $[15,30]$. Canine periodontitis is almost identical to periodontitis in humans in terms of disease course, primary pathogens and clinical presentation $[18,19]$. The causative microbial agent, Porphyromonas gingivalis, tends to be detected around 6-12 months old in dogs, and also gingivitis starts to be found around these ages. By age 5, the percentage of Porphyromonas spp. isolated from canine plaque is roughly 6-fold higher than that at 2 years of age, and correlates with disease severity [15].

In this way, LDOA of IFN- $\alpha$ can be used as safe and simple way of protection and therapeutics against periodontal disease. We considered that naturally occurred canine periodontal disease can be used as a model disease to examine the efficacy of LDOA of IFN- $\alpha$. Since canine IFN- $\alpha$ $(\mathrm{CaIFN}-\alpha)$ was cloned and expressed by Himmler et al. [16], 8 subtypes of CaIFN- $\alpha$ have been reported to the GenomeNet database service and 5 of these 8 subtypes have been cloned, expressed, and characterized in Escherichia coli [35]. To obtain further information on the biological activities of CaIFN- $\alpha$, Ruttanapumma et al. [31] reported the expression and purification of bioactive CaIFN- $\alpha 4$ using baculovirus expression system. In this study, we examined the efficacy of orally administered CaIFN- $\alpha 4$ against naturally occurring canine periodontal disease, especially gingival inflammation. 


\section{MATERIALS AND METHODS}

Preparation of CaIFN- $\alpha 4$ : The CaIFN- $\alpha 4$ was kindly provided from Laboratory of Zoonosis, School of Veterinary Medicine, Kitasato University, Towada, Aomori, Japan. The CaIFN- $\alpha 4$ was dissolved in phosphate buffered saline ( $\mathrm{pH}$ 7.0). Its purity was confirmed by sodium dodecyl sulphate-polyacrylamide gel electrophoresis and Coomassie Brilliant Blue staining. The quantity of the purified CaIFN$\alpha 4$ was estimated to be approximately $200 \mu \mathrm{g} / \mathrm{ml}$. The antiviral activity of CaIFN- $\alpha 4$ was determined by cytopathic effect (CPE) inhibition assay using Vesicular stomatitis virus (VSV) on the canine fibroma cell line A72 [31]. The IFN titer, expressed in laboratory units per $\mathrm{m} l(\mathrm{LU} / \mathrm{m} l)$, was calculated as a reciprocal of dilution of the wells in which $50 \%$ of the monolayer was protected from virus-induced CPE. The titer of CaIFN- $\alpha 4$ was estimated to be $1024 \times 10^{4}$ $\mathrm{LU} / \mathrm{m} l$. The specific activity of CaIFN- $\alpha 4$ on A72 cells was calculated to be approximately $5.0 \times 10^{4} \mathrm{LU} / \mu \mathrm{g}$ protein.

Low-dose oral administration of CaIFN- $\alpha 4$ : It is reported that there is an appropriate dose for LDOA of IFN- $\alpha$ and the lower dosages of IFN- $\alpha$ in the LDOA range show greater effects [6]. We employed two kinds of administration dosages: $0.25 \mathrm{LU} / \mathrm{kg}$ and $25 \mathrm{LU} / \mathrm{kg}$, which are in the range of LDOA, to examine and obtain some information on appropriate doses of CaIFN- $\alpha 4$ for LDOA. CaIFN- $\alpha 4$ at a dose of 0.25 or $25 \mathrm{LU} / \mathrm{kg} /$ day was administered once daily in paste form with maltose into the oral cavities of the dogs. Maltose was added to improve storage stability of IFN- $\alpha 4$ [40]. All dogs were treated every day. Before CaIFN- $\alpha 4$ treatment, maltose paste without CaIFN- $\alpha 4$ was administered to Group 1.

Animals: A total of 29 beagle dogs were divided into three groups: Group 1, Group2, and Group 3. Group 1 was consisted of 5 dogs without gingival inflammation (8-9 months of age, body weight 9-10 kg, females) and treated with CaIFN- $\alpha 4$ for 5 days. Group 2 was consisted of 12 dogs with gingival inflammation (15 months of age, body weight $9-13 \mathrm{~kg}$, females). These dogs were further divided into two subgroups, a CaIFN- $\alpha 4$ subgroup of 6 dogs and a control subgroup of the other 6 dogs, and treated for 7 days with CaIFN- $\alpha 4$ and maltose paste without CaIFN- $\alpha 4$, respectively. Group 3 was consisted of 12 dogs with gingival inflammation (17 months of age, body weight $10-13 \mathrm{~kg}$, females), Group 3 dogs were divided into three subgroups, a CaIFN- $\alpha 4(0.25 \mathrm{LU} / \mathrm{kg})$ subgroup of $4 \mathrm{dogs}$, a CaIFN- $\alpha 4$ $(25 \mathrm{LU} / \mathrm{kg})$ subgroup of another $4 \mathrm{dogs}$, and a control subgroup of the remaining $4 \mathrm{dogs}$, and treated for 30 days with CaIFN- $\alpha 4(0.25 \mathrm{LU} / \mathrm{kg}), \mathrm{CaIFN}-\alpha 4(25 \mathrm{LU} / \mathrm{kg})$ and maltose paste without CaIFN- $\alpha 4$, respectively. The dogs were obtained from Kitayama Labes Co., Ltd. (Yamaguchi, Japan). The animal experimental protocol was approved by the Animal Care and Use Committee of the Kitasato Institute, and the experiments were performed according to their standards.

Gingival index: The degree of gingival inflammation was estimated according to the criteria of the gingival index (GI) system described by Löe and Silness [23] with some modification, and the degree of gingival inflammation was classified into 4 ranks as follows: 0 , healthy gingiva without gingivitis; 0.5 , gingivitis with slight inflammation in the form of a dotted line; 1 , gingivitis with inflammation in the form of a clear red line; 2 , gingivitis with inflammation in the form of a clear red line accompanied by hemorrhaging. GI was examined only on the buccal aspects of all teeth. GI was determined by the way shown above, and changes of GI were evaluated as percentage comparison where the GI determined just before LDOA in each group is $100 \%$. Also, occult blood was tested as a clinical sign of gingival inflammation, using a paper test kit for oral occult blood (Showa Yakuhin Co., Ltd., Tokyo, Japan).

Determination of salivary 8-hydroxydeoxyguanosine and $N \varepsilon$-(hexanoyl) lysine by enzyme-linked immunosorbent assay: The saliva samples of the dogs in Group 1 were centrifuged at $10,000 \times \mathrm{g}$ for $10 \mathrm{~min}$, and the supernatant was used to determine 8-hydroxydeoxyguanosine ( $8-\mathrm{OHdG})$ and $\mathrm{N} \varepsilon$-(Hexanoyl) Lysine (HEL) levels as oxidative stress biomarkers [36, 20] with enzyme-linked immunosorbent assay (ELISA) kits (Japan Institute for the Control of Aging, Nikken SEIL Corporation, Fukuroi, Shizuoka, Japan).

8 -OHdG is a product of oxidatively damaged DNA formed by hydroxy radical, singlet oxygen and direct photodynamic action. 8-OHdG can be detected in tissue, serum, urine and other biomaterials in the ELISA kit utilising monoclonal antibody which is highly specific for DNA damage [32]. Oxidative damage of lipids caused by reactive oxygen species (ROS) plays an important role in inflammatory diseases. The HEL is one of the biomarkers for oxidative stress [21]. The HEL moiety is generated from the reaction of lipid hydroperoxidase with protein and can be detected by the ELISA kit.

Examination of the genus Porphyromonas: The saliva was taken from each of the 5 dogs in Group 1 and put into sterilized anaerobic tubes with brain heart infusion (BHI) broth (Nissui Co., Tokyo, Japan), and these tubes were placed in anaerobic glove boxes (Hirasawa Co., Tokyo, Japan) at the condition of $70 \% \mathrm{~N}_{2}, 15 \% \mathrm{CO}_{2}$ and $15 \% \mathrm{H}_{2}$ [18]. The diluted suspension of each sample was placed on BHI agar (Nissui) with 7\% horse blood in order to count Porphyromonas as black-pigmented bacteria. Identification of representative colonies was carried out using the API $20 \mathrm{~A}$ and API-Zyme systems (API Systems, Montalieu, France). The Porphyromonas count was calculated from the number of colonies multiplied by the dilution factor, and the percentage of dogs with Black-Pigmented Porphyromonas (BPP) was then calculated.

Statistical analysis: Significant differences between the means of the groups were determined by Mann-Whitney $\mathrm{U}$ test in Fig. 1, and the unpaired $t$-test in Figs. 2 A, 2 B, Fig. 3 , and 4 . Probability values of $<0.05$ were considered significant. 


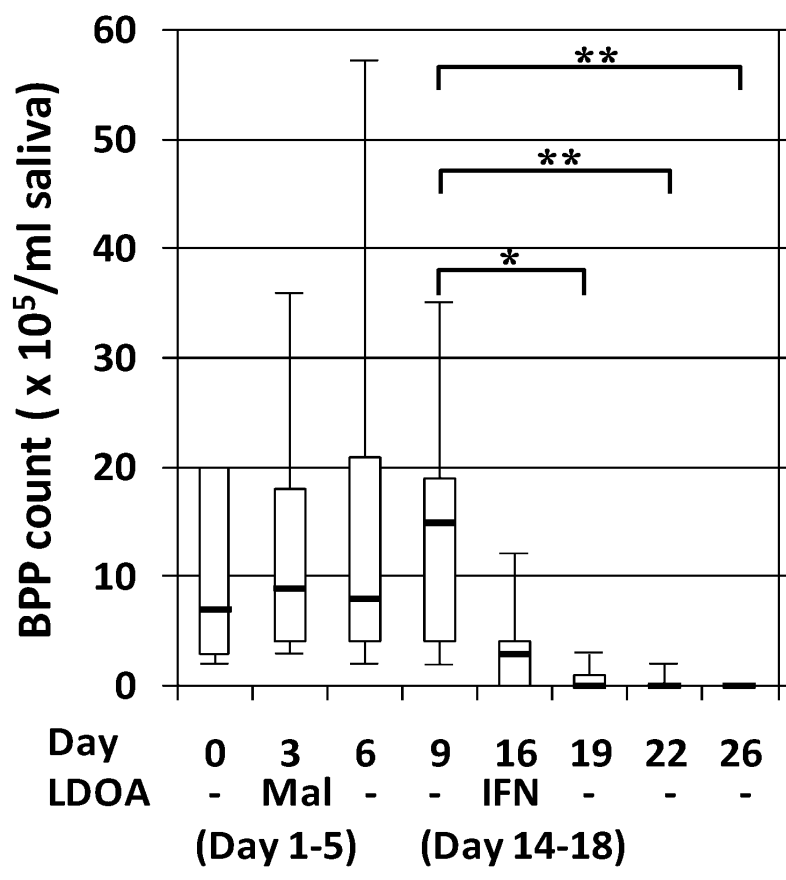

Fig. 1. Ability of orally administered CaIFN- $\alpha 4$ to inhibit BPP. Group 1 dogs were administered maltose paste containing CaIFN- $\alpha 4(0.25 \mathrm{LU} / \mathrm{kg})$ daily for 5 days (from day 14 to day 18 ) in the oral cavity. Before CaIFN- $\alpha 4$ administration, the dogs received maltose paste without CaIFN- $\alpha 4$ (Mal) administered in the same manner for 5 days (from day 1 to day 5 ). A saliva sample was taken from each dog on day $0,3,6,9,16,19$, 22 and 26 to isolate BPP. The boxes show the interquartile range (between 25th and 75th percentiles), and the horizontal lines within the boxes show the median. The whisker caps enclose the values between the minimum and the maximum. * $\mathrm{P}<0.05 ; * * \mathrm{P}<0.01$, determined by the Mann-Whitney U-test.

\section{RESULTS}

Ability of orally administered CaIFN- $\alpha 4$ to inhibit naturally occurring Porphyromonas infection: The ability of orally administered CaIFN- $\alpha 4$ to inhibit naturally occurring Porphyromonas infection was examined by treating each dog in Group 1 daily with $0.25 \mathrm{LU} / \mathrm{kg}$ CaIFN- $\alpha 4$.

Before CaIFN- $\alpha 4$ administration was initiated, the dogs were treated daily with maltose paste without CaIFN- $\alpha 4$. The dogs treated with only maltose paste from day 1 to day 5 and maltose paste with CaIFN- $\alpha 4$ from day 14 to day 18 during the experiment. Saliva was taken from the dogs on day $0,3,6,9,16,19,22$ and 26 during the experiment. BPP, occult blood, HEL, and 8-OHdG were examined using these saliva samples. BPP was isolated from all dogs when they were examined before the experiment. When maltose paste without CaIFN- $\alpha 4$ was administered, the number of dogs with BPP remained constant, but when the dogs were treated daily for 5 days with $0.25 \mathrm{LU} / \mathrm{kg} \mathrm{CaIFN-} \alpha 4$, the number of dogs with BPP was reduced by day 16 and was reduced by increasingly greater percentages on day 19,22 , and 26 . On day 26, after 8 days from the end of CaIFN- $\alpha 4$ treatment, we were unable to isolate Porphyromonas from the saliva. Figure 1 shows the BPP count in the saliva of each dog. A decrease in the number of BPP was observed after 2 days of the CaIFN- $\alpha 4$ treatment. In contrast, BPP remained constant during treatment with maltose paste without CaIFN$\alpha 4$.

Reduction of the stress response after the administration of CaIFN- $\alpha 4$ : The mean HEL concentration in the saliva of the dogs in Group 1 (Fig. 2A) before the experiments were $33.8 \pm 19.0 \mathrm{ng} / \mathrm{m} l$ (Before Mal), and 35.1 $\pm 17.1 \mathrm{ng} / \mathrm{ml}$ (Mal). There was no significant difference between Before Mal group and Mal control group. After LDOA of CaIFN$\alpha 4$, the level decreased significantly $16.2 \pm 8.0(\mathrm{P}<0.05)$. The level of salivary $8-\mathrm{OHdG}$ also decreased significantly after LDOA of CaIFN- $\alpha 4$ (MAL : $5.6 \pm 2.6 \mathrm{ng} / \mathrm{ml}$, IFN : 2.4 $\pm 2.8 \mathrm{ng} / \mathrm{ml}, \mathrm{P}<0.01$, Fig. 2B). There was no significant difference between Before Mal $(4.9 \pm 2.4 \mathrm{ng} / \mathrm{ml})$ group and Mal group.

Reduction of gingival inflammation after the administration of CaIFN- $\alpha 4$ : In the Group 2 dogs, gingival inflammation was observed both before and after the experiment. LDOA of CaIFN- $\alpha 4$ was found to be effective in treating canine gingivitis (Fig. 3). Compared to the control subgroup, the CaIFN- $\alpha 4$ subgroup of Group 2 showed significantly low GI $(\mathrm{P}<0.05)$. On day 0 (the beginning of LDOA of CaIFN- $\alpha 4$ ), the GIs were $0.62 \pm 0.04$ in the CaIFN- $\alpha 4$ subgroup and $0.68 \pm 0.12$ in the control subgroup, and this difference was not statistically significant. The dogs were treated daily with CaIFN- $\alpha 4$ for 7 days and gingival inflammation was examined. Compared to the GI on day 0 relatively, the relative GI of CaIFN administered group on day 6 declined to $72.7 \pm 10.3 \%(\mathrm{GI}=0.45 \pm 0.08)$, but that of Control group on day 6 rose to $109.8 \pm 11.5 \%$, which was statistically significant $(\mathrm{P}<0.01)$. Additionally, maltose alone (control subgroup of Group 2) showed no effect on GI.

Moreover, in order to examine if the long-term LDOA of CaIFN- $\alpha 4$ shows continuous effect on improving gingival inflammation, we examined group 3 dogs (17 months of age), in which gingival inflammation was observed both before and after the experiment. On day -1 (the day before the beginning of LDOA), GIs were $0.84 \pm 0.09$ in the control subgroup, $0.85 \pm 0.09$ in the $0.25 \mathrm{LU} / \mathrm{kg}$ subgroup, and 0.76 \pm 0.12 in the $25 \mathrm{LU} / \mathrm{kg}$ subgroup. There was no significant difference among each group. Compared to the GI on day 1 relatively, the relative GI values on day 7 declined to 74.2 $\pm 4.6 \%(\mathrm{GI}=0.63 \pm 0.10)$ in $0.25 \mathrm{LU} / \mathrm{kg}$ group and $72.9 \pm$ $5.2 \%(\mathrm{GI}=0.56 \pm 0.12)$ in $25 \mathrm{LU} / \mathrm{kg}$ group, which was statistically significant. Moreover, on day 30 the relative GI values declined to $63.6 \pm 11.1 \%(\mathrm{GI}=0.55 \pm 0.14)$ in $0.25 \mathrm{LU} /$ $\mathrm{kg}$ group and $62.6 \pm 12.2 \%(\mathrm{GI}=0.48 \pm 0.14)$ in $25 \mathrm{LU} / \mathrm{kg}$ group, which was also statistically significant.

\section{DISCUSSION}

In the present study, we examined the ability of orally administered CaIFN- $\alpha 4$ to protect against canine periodon- 
A

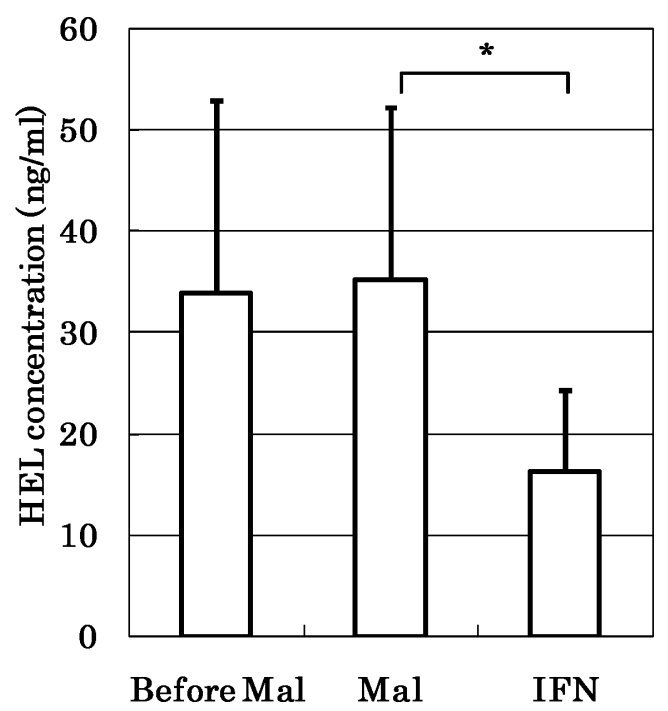

B

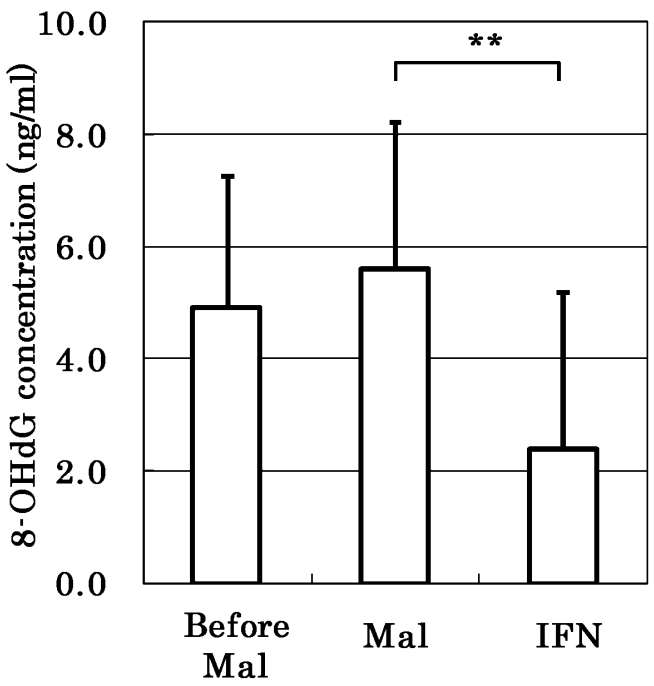

Fig. 2. Changes in the oxidative stress markers HEL and 8-OHdG in Group 1 dogs after LDOA of CaIFN- $\alpha 4$. A saliva sample was taken from each of the $5 \mathrm{dogs}$ in Group 1 before Mal administration (corresponds to the point of day 0 in Fig. 1), after Mal administration (corresponds to the point of day 9 in Fig. 1) and after CaIFN- $\alpha 4$ administration (corresponds to the point of day 26 in Fig.1) to determine the concentrations of HEL (A) and 8-OHdG (B). Each bar represents mean $\pm \mathrm{SD}(\mathrm{n}=5)$. *, **, significantly different from after Mal values $(* \mathrm{P}<0.05 ; * * \mathrm{P}<0.01)$.

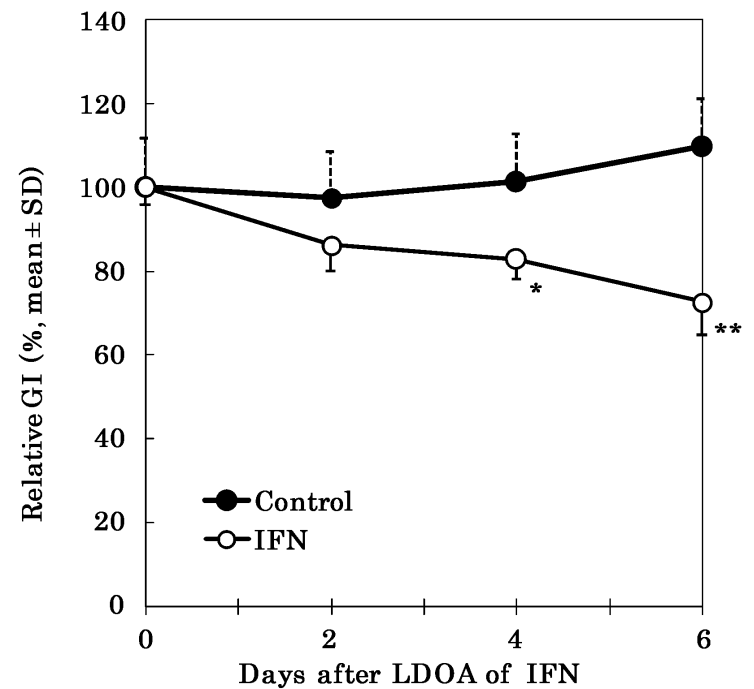

Fig. 3. Comparison of relative GI before and after LDOA of CaIFN- $\alpha 4$ in Group 2. Group 2 dogs were treated daily for 7 days with maltose paste with $(\bigcirc)$ or without $(O)$ CaIFN- $\alpha 4$ $(0.25 \mathrm{LU} / \mathrm{kg})$. GI was examined before and after CaIFN- $\alpha 4$ administration. Data are shown as relative value to day 0. Data are mean $\pm \mathrm{SD}(\mathrm{n}=6$ in each subgroup). *, **, significantly different from control values $(* \mathrm{P}<0.05 ; * * \mathrm{P}<0.01)$.

tal disease. Daily LDOA of canine recombinant CaIFN- $\alpha 4$ expressed in a baculovirus system $(0.25 \mathrm{LU} / \mathrm{kg})$ was found to reduce periodontopathic bacterial counts, stress

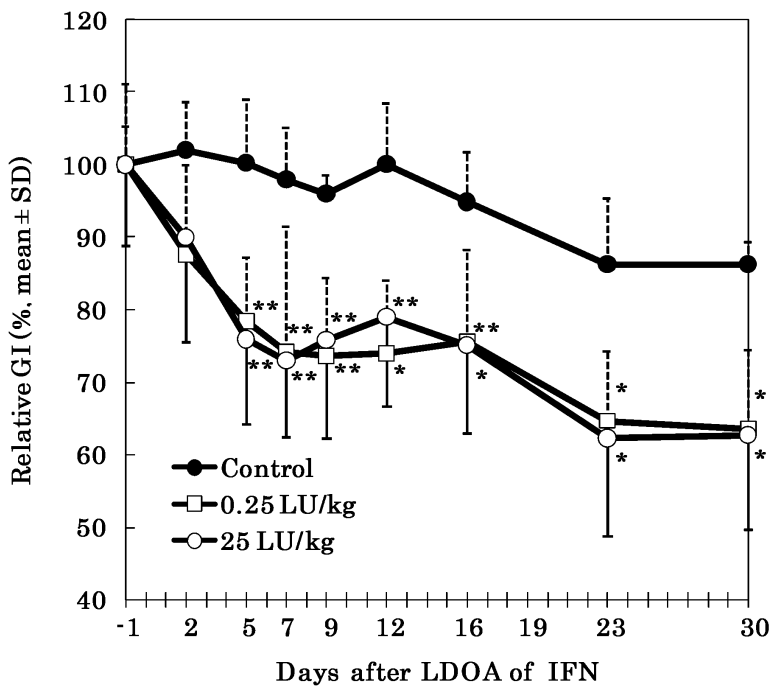

Fig. 4. Comparison of relative GI before and after LDOA of CaIFN- $\alpha 4$ in Group 3. Group 3 dogs were treated daily for 30 days with maltose paste with $(\square, 0.25 \mathrm{LU} / \mathrm{kg} ; \bigcirc, 25 \mathrm{LU} / \mathrm{kg})$ or without (O) CaIFN- $\alpha 4$. GI was examined before and after CaIFN- $\alpha 4$ administration. Data are shown as relative value to day -1 . Data are mean $\pm \mathrm{SD}(\mathrm{n}=4$ in each subgroup). *,**, significantly different from control values $(* \mathrm{P}<0.05$; ** $\mathrm{P}<0.01)$.

responses, and gingival inflammation. Our observations indicate that recombinant CaIFN- $\alpha 4$ can enhance defense responses in canine periodontal disease. 
Low dose administration of IFN- $\alpha$ is defined as IFN- $\alpha$ administration with the amount of $0.05-1000 \mathrm{IU} / \mathrm{kg}$, compared to high dose administration of IFN- $\alpha$, which is one of the traditional treatments by intramuscular or subcutaneous injection applied to hepatitis $\mathrm{C}$, uses several hundreds to ten million IU/kg [39]. Human IFN- $\alpha$ (HuIFN- $\alpha$ ) has been given orally in low doses to treat or prevent a number of infectious and immune-based diseases in a variety of species $[2,7,14,26]$. Notably, the oral administration of natural HuIFN- $\alpha$ has been shown to be therapeutic in respiratory tract infections in cattle $[6,13]$. What is important is there is a proper amount of IFN- $\alpha$ for low dose administration, and it is reported that smaller amounts of substance tends to show greater effects [6]. In this study two ways of IFN- $\alpha$ administration were employed: $0.25 \mathrm{LU} / \mathrm{kg}$ and $25 \mathrm{LU} / \mathrm{kg}$, both of which are in the range of low dose administration and showed almost the same effects. The mechanism of how orally administered IFN- $\alpha$ influences the systemic/ local immune system remains unclear. It is well accepted that type I interferons play a critical role in modulating immune responses to various antigens $[3,4]$.

It has been estimated that more than 400 bacterial species reside in the oral cavity [28]. Some of these cause inflammation (e.g., periodontitis), and understanding of this condition requires examination of the innate immunity of the oral cavity. Oral mucosal cells such as epithelial cells are thought to act as a physical barrier against the invasion of pathogenic organisms, but have the ability to produce inflammatory cytokines and express adhesion molecules [34]. Sakura et al. [33] suggest that IFNs might be capable of both enhancing and inhibiting inflammatory responses in periodontal tissues through the dual regulation of IL-8 production by gingival fibroblasts in response to bacterial components and cytokines. Therefore, we assume there was a possibility that these systems have some relations to the mismatch of the time on observable significant differences between decrease of BPP counts (Group 1) and reduction of GI (Group 2) in this study.

Recently, several studies have shown the expression of $\beta$ defensins, positively charged antimicrobial peptides, in inflammatory gingival diseases $[8,10,22]$. The observed decrease in the BPP count in canine saliva in the present study might be due to antimicrobial peptides such as $\beta$ defensin induced by the LDOA of CaIFN- $\alpha 4$.

In the present study, we evaluated the levels of $8-\mathrm{OHdG}$ [36] and HEL [20], which are both oxidative stress markers, in whole saliva as biomarkers in clinically healthy individuals (Group 1). Both 8-OHdG and HEL decreased significantly after LDOA of CaIFN- $\alpha 4$ In Group 1, 4 of 5 dogs tested positive for occult blood, but this rate decreased during LDOA of CaIFN- $\alpha 4$ (data not shown), possibly reflecting stress levels. Recently, Takane et al. [36] reported that $8-\mathrm{OHdG}$ levels in saliva can reflect the status of periodontal health, because eliminating inflammation of the periodontium would logically lead to a decrease in $8-\mathrm{OHdG}$. Our observation that the HEL and $8-\mathrm{OHdG}$ levels in saliva were reduced by LDOA of CaIFN- $\alpha 4$ is consistent with this report.

In this Group 1 experiment we used saliva of examined dogs as testing samples. However, we could hardly arrange a control group other than CaIFN- $\alpha 4$ group because there was a large variation in the amount of individual saliva samples from dogs, and thus it was very difficult to prepare dogs yielding enough saliva for the study. Therefore, we had maltose-administered dogs treated CaIFN- $\alpha 4$ after maltose washout period, and then we compared the data maltoseadministered and CaIFN- $\alpha 4$-administered periods.

We carried out Group 2 and Group 3 experiments as Group 1, in which we examined BPP, HEL, and 8-OHdG with saliva sample. However, the results didn't show any significant differences because of large variations among individual samples (data not shown). We assume this is because of variations in volume of saliva samples in examined dogs, which caused difficulty in obtaining enough data for analysis. Generally it is difficult to obtain enough volume of saliva samples from dogs [9]; however, we may be able to improve the method collecting saliva from dogs with some kinds of stimulants without any effects on detecting enzymes so that we can obtain enough amounts of saliva samples and more data to reproduce Group 1 results.

This study showed that LDOA of CaIFN- $\alpha 4$ reduces gingival inflammation, in an effect that may be associated with the inhibition of bacterial growth. Furthermore, our examination indicates that the anti-inflammatory effect of LDOA of CaIFN- $\alpha 4$ could continue for as long as 30 days with continuous LDOA of CaIFN- $\alpha 4$ with a little amount such as 25 $\mathrm{LU} / \mathrm{kg}$, or $0.25 \mathrm{LU} / \mathrm{kg}$ which gave us similar results to 25 $\mathrm{LU} / \mathrm{kg}$. In addition to this, in Fig. 3, the GI value at the starting point is lower than that in Fig. 4, and this is because we used young dogs with mild inflammation at the starting point.

Periodontal diseases, which cause the loss of teeth, are one of the predominant disorders in the oral cavities of various animals. The present study implied LDOA of IFN- $\alpha$ can be a new way of protection and treatment against periodontal disease in mammals including human. Likewise in dogs, it has been estimated that approximately $80 \%$ of dogs demonstrate some degree of periodontal disease by 4 years of age. Currently, the clinical control of canine periodontitis relies largely on antibiotics, but this may cause the emergence of antibiotic-resistant bacteria. The present results suggest that the clinical application of LDOA of IFN- $\alpha$ may protect dogs from canine periodontal diseases with no side effects, which would enhance the quality of life of the dogs. In addition, the clinical use of IFN- $\alpha$ for periodontal diseases may be effective not only for dogs but also for humans, because canine periodontitis is almost identical to periodontitis in humans.

ACKNOWLEDGMENTS. This work was supported in part by a grant from the New Energy and Industrial Technology Development Organization. The authors would like to thank Dr. Kazuaki Takehara for purified recombinant CaIFN- $\alpha 4$ expressed in a baculovirus system. 


\section{REFERENCES}

1. Baron, S., Coppenhaver, D.H., Dianzani, F., Fleischman, W.R., Hugh, T.K., Klimpel, D.R., Niesel, D.W., Stanton, G.J. and Tyring, S.K. 1992. Principles and medical applications. pp. 115. In: Interferon (Baron, S. ed.), The University of Texas at Galveston, Galveston.

2. Beilharz, M.W., Cummins, J.M. and Bennett, A.L. 2007. Protection from lethal influenza virus challenge by oral type 1 interferon. Biochem. Biophys. Res. Commun. 355: 740-744.

3. Belardelli, F. and Gresser, I. 1996. The neglected role of type I interferon in the T-cell response: implications for its clinical use. Immunol. Today 17: 369-372.

4. Brassard, D.L., Grace, M.J. and Bordens, R.W. 2002. Interferon-alpha as an immunotherapeutic protein. J. Leukocyte Biol. 71: 565-581.

5. Brod, S.A., Khan, M., Kerman, R.H. and Pappolla, M. 1995. Oral administration of human or murine interferon alpha suppresses relapses and modifies adoptive transfer in experimental autoimmune encephalomyelitis. J. Neuroimmunol. 58: 61-69.

6. Cummins, J.M., Hutcheson, D.P., Georgiades, J. A. and Richard, A.B. 1993. Oral therapy with human interferon alpha in calves experimentally injected with infectious bovine rhinotracheitis virus. Arch. Immunol. Ther. Exp. 41: 193-198.

7. Cummins, M.J., Papas, A., Kammer, G.M. and Fox, P.C. 2003. Treatment of primary Sjögren's syndrome with low-dose human interferon alpha administered by the oromucosal route: combined phase III results. Arthritis Rheum. 49: 585-593.

8. Dommisch, H., Açil, Y., Dunsche, A., Winter, J. and Jepsen, S. 2005. Differential gene expression of human beta-defensins (hBD-1, -2, -3) in inflammatory gingival diseases. Oral Microbiol. Immunol. 20: 186-190.

9. Dreschel, N.A. and Granger, D.A. 2009. Methods of collection for salivary cortisol measurement in dogs. Horm. Behav. 55: 163-168.

10. Eberhard, J., Menzel, N., Dommisch, H., Winter, J., Jepsen, S. and Mutters, R. 2008. The stage of native biofilm formation determines the gene expression of human $\beta$-defensin-2, psoriasin, ribonuclease 7 , and inflammatory mediators: a novel approach for stimulation of keratinocytes with in situ formed biofilms. Oral Microbiol. Immunol. 23: 21-28.

11. Fleischmann, W.R. Jr., Koren, S. and Fleischmann, C.M. 1992. Orally administered interferons exert their white blood cell suppressive effects via a novel mechanism. Proc. Soc. Exp. Biol. Med. 201: 200-207.

12. Fujihashi, K., Kono, Y., Beagley, K.W., Yamamoto, M., McGhee, J.R., Mestecky, J. and Kiyono, H. 1993. Cytokine and periodontal disease: immunopathological role of interleukins for B cell responses in chronic inflamed gingival tissues. J. Periodontol. 64: 400-406.

13. Georgiades, J.A. 1993. Effect of low dose natural human interferon alpha given into the oral cavity on the recovery time and death loss in feedlot hospital pen cattle: a field study (short communication). Arch. Immunol. Ther. Exp. 41: 205-208.

14. Gilger, B.C., Rose, P.D., Davidson, M. G., Roberts, S. M. and Miller, T. 1999. Low-dose oral administration of interferonalpha for the treatment of immune-mediated keratoconjunctivitis sicca in dogs. J. Interferon Cytokine Res. 19: 901-905.

15. Harvey, C.E. and Emily, P.P. 1993. Hyperplastic gingivitis. pp. 104. In: Small Animal Dentistry (Harvey, C.E. and Eimily, P.P. eds.), Mosvy-Year Books, St. Louis.

16. Himmler, A., Hauptmann, R., Adolf, G.R. and Swetly, P. 1987. Structure and Expression in Escherichia coli of Canine Inter- feron- $\alpha$ Genes. J. Interferon Res. 7: 173-183.

17. Hori, T., Katafuchi, T., Take, S. and Shimizu, N. 1998. Neuroimmunomodulatory actions of hypothalamic interferonalpha. Neuroimmunomodulation 5: 172-177.

18. Isogai, H., Isogai, E., Okamoto, H., Shirakawa, F., Nakamura, T., Matsumoto, T., Watanabe, T., Miura, H., Aoi, Y., Kagota, W. and Takano, K. 1989. Epidemiological study on periodontal diseases and some other dental disorder in dogs. Jpn. J. Vet. Sci. 51: 1151-1162.

19. Isogai, H., Kosaka, Y., Benno, Y. and Isogai, E. 1999. Ecology of genus Porphyromonas in canine periodontal disease. J. Vet. Med. B 46: 467-473.

20. Kato, Y., Mori, Y., Makino, Y., Morimitsu, Y., Hiroi, S., Ishikawa, T. and Osawa, T. 1999. Formation of Ne-(hexanonyl) lysine in protein exposed to lipid hydroperoxide. J. Biol. Chem. 274: 20406-20414.

21. Kato, Y., Miyake, Y., Yamamoto, K., Shimomura, Y., Ochi, H., Mori, Y. and Osawa, T. 2000. Preparation of a monoclonal antibody to N(epsilon)-(Hexanonyl)lysine: application to the evaluation of protective effects of flavonoid supplementation against exercise-induced oxidative stress in rat skeletal muscle. Biochem Biophys Res. Commun. 274: 389-393.

22. Krisanaprakornkit, S., Weinberg, A., Perez, C.N. and Dale, B.A. 1998. Expression of the peptide antibiotic human betadefensin 1 in cultured gingival epithelial cells and gingival tissues. Infect. Immun. 66: 4222-4228.

23. Löe, H. and Silness, J. 1963. Periodontal disease in pregnancy, I. prevalence and severity. Acta Odontol. Scand. 21: 533-551.

24. Loesche, W.J. and Grossman, N.S. 2001. Periodontal disease as a specific, albeit chronic infection: diagnosis and treatment. Clin. Microb. Rev. 14: 727-752.

25. Mathur, A., Michalowicz, B., Castillo, M. and Aeppli, D. 1996. Interleukin-1 alpha, interleukin-8 and interferon-alpha levels in gingival crevicular fluid. J. Periodontal Res. 31: 489-495.

26. Ohya, K., Matsumura, T., Itchoda, N., Ohashi, K., Onuma, M. and Sugimoto, C. 2005. Ability of orally administered IFN$\alpha$-containing transgenic potato extracts to inhibit Listeria monocytogenes infection. J. Interferon Cytokine Res. 25: 459466.

27. Page, R.C. 1991. The role of inflammatory mediators in the pathogenesis of periodontal disease. J. Periodontal. Res. 26: 230-242.

28. Paster, B.J., Boches, S.K., Galvin, J.L., Ericson, R.E., Lau, C.N., Levanos, V.A., Sahasrabudhe, A. and Dewhirst, F.E. 2001. Bacterial diversity in human subgingival plaque. J. Med. Microbiol. 183: 3770-3783.

29. Paul, W.E. and Seder, R.A. 1994. Lymphocyte responses and cytokines. Cell 76: 241-251.

30. Penman, S. and Harvey, C.E. 1995. Periodontal disease. pp. 37-39. In: Manual of Small Animal Dentistry (Harvey, C.E., Orr, H.D. eds.), B. S. A. V. A., Cheltenham.

31. Ruttanapumma, R., Anzai, M., Takegawa, M., Okamura, M., Maehara, N., Sato, K., Yoshioka, K., Itoh, A., Nakamura, M. and Takehara, K. 2006. High level expression of biologically active canine interferon- $\alpha$ subtype 4 using a baculovirus. $J$. Vet. Med. Sci. 68: 1347-1349.

32. Saito, S., Yamauchi, H., Hasui, Y., Kurashige, J., Ochi, H. and Yoshida, K. 2000. Quantitative determination of urinary 8hydroxydeoxyguanosine (8-OHdG) by using ELISA. Res. Commun Mol. Pathol. Pharmacol. 107: 39-44.

33. Sakura, T., Tokuda, M., Tamura, M., Jimi, E., Ikebe, T., Koba, T., Nagaoka, S. and Takada, H. 1998. Dual regulatory effects of interferon-alpha, -beta, and -gamma on interleukin- 8 gene 
expression by human gingival fibroblasts in culture upon stimulation with lipopolysaccharide from Prevotella intermedia, interleukin-1 alpha, or tumor necrosis factor-alpha. J. Dent. Res. 77: 1597-1605.

34. Sugawara, S., Uehara, A., Tamai, R. and Takada, H. 2002. Innate immune responses in oral mucosa. J. Endotoxin Res. 8: 465-468.

35. Taira, O., Watanugi, I., Hagiwara, Y., Takahashi, M., Arai, S., Sato, H. and Maehara, N. 2005. Cloning and expression of canine interferon-alpha genes in Escherichia coli. J. Vet. Med. Sci. 67: 1059-1062.

36. Takane, M., Sugano, N., Ezawa, T., Uchiyama, T. and Ito, K. 2005. A marker of oxidative stress in saliva: association with periodontally-involved teeth of a hopeless prognosis. J. Oral Sci. 47: 53-57.

37. Tompkins, W.A. 1999. Immunomodulation and therapeutic effects of the oral use of interferon-alpha: mechanism of action. J. Interferon Cytokine Res. 19: 817-828.

38. Tovey, M.G., Meritet, J.F., Guymarho, J. and Maury, C. 1999. Mucosal cytokine therapy: marked antiviral and antitumor activity. J. Interferon Cytokine Res. 19: 911-921.

39. Witthöft, T. 2008. Review of consensus interferon in the treatment of chronic hepatitis C. Biologics. 2: 635-643.

40. Yamaguchi, H., Tatefuji, T., Ikeda, M., Nanba, M., Seno, H., Ando, S., Sato, Y. and Kurimoto, M. 1995. Prolonged stability of natural human interferon alpha (IFN- $\alpha$ ) lozenges using anhydrous maltose as an excipient. YAKUZAIGAKU 55: 7580.

41. Yoshino, S. 1995. The preventive effect of oral administration of type I interferon on collagen-induced arthritis in rats. Exp. Mol. Pathol. 62: 123-130. 